\title{
Influence du rayonnement des gaz dans les calculs d'échangeurs de chaleur
}

\section{Gas radiation effects on heat exchanger design}

\author{
PAR \\ M. VIGNERON \\ $\mathrm{ET}$ \\ M. STOLY

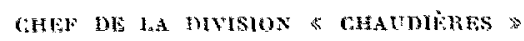 \\ SERVICE DE TA PROOUCTION THERMLUE D'ÉLEGTHCCTE DE FHANGE
}

\begin{abstract}
Libhide de re memoire esef de montrer dotns

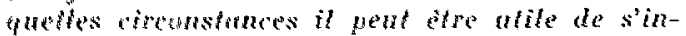

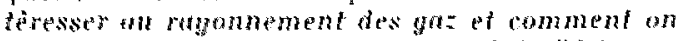
peut th tenir comple lors du cotoul de labhange de chulenr, fess antears expensent succinctenent

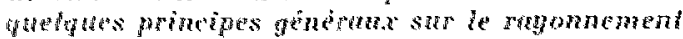

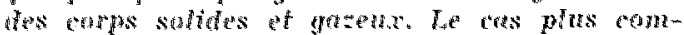

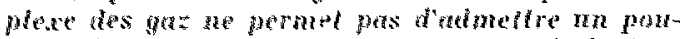

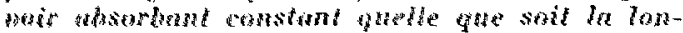

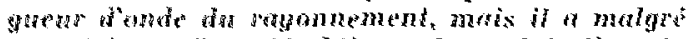

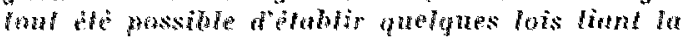

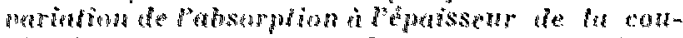

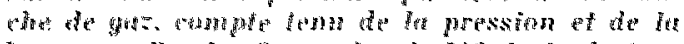

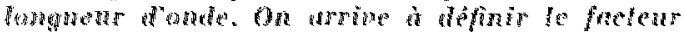

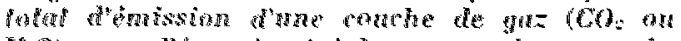

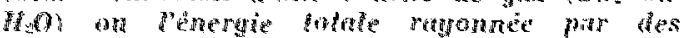

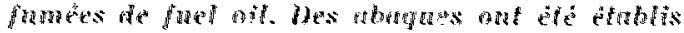

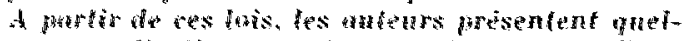

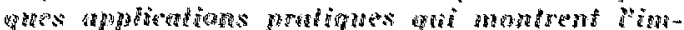

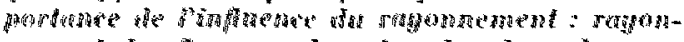

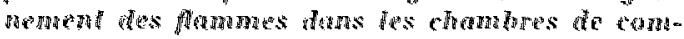

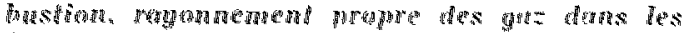

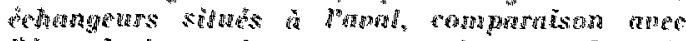

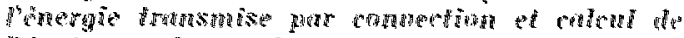

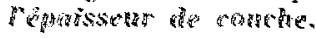

\begin{abstract}
This paper is intended to show in what circmstunces it maty be useful to concern one sell with the radiation from gases and how this can be taken into account in heat transier calculations. The athors give a succinct accomm of a few of the general principles of radiations. from solid hodies and guses. Gases, which are the more complicated of the two, cunnot be said to have constant absorbant power whatever the wavelength of the radiation may be. But in spife of this we can derive laws connecting variation in absorption with the thichness of the laper of gas faking into consideration the pressure and the mane length. Thus we an define to fotal emission factor of a layer of gess $\left(\mathrm{CO}_{3}\right.$ or $\mathrm{H}_{3} \mathrm{O}$ ) or the tolal radiated energy of diesel oil fumes. Charts have been drann based on these assumptions. The anthors use these laws to give practionl applications showing the importance of matation infuence. Such applications are radiation from fames in combustion chombers. the radiation of gases in heat exchangers betow such combustion chambers, a comparison anth

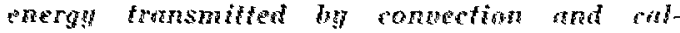
woting the thicliness of the layer.
\end{abstract}

\section{I. - INTRODUCTION}

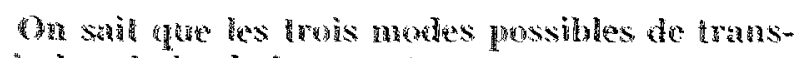

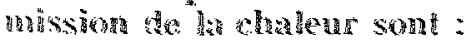

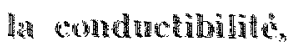

In conver tor

(1)

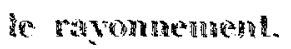

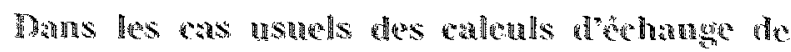

chaleur, lun de ces trois modes esl en gerueral pepondém, el retient lout dabord lattenlium. Cependant, il est très rave que l'on puisse ressoudre eompletement un problime de transmission de chaleur sans tenir compte des divers modos capables dintervenir.

Par example on ne peut gurite ellutier la dis- 
tribution des températures et des flux de chaleur dans un élément d'ailettage. problème de conductibilité, si l'on ne connaît pas, par ailleurs, les conditions d'échange à la surface limitant cette ailette, conditions habituellement liées à la convection forcée d'un fluide circulant le long de cette surface. De même, lors du calcul d'un échangeur à deux fluides, il peut être utile de tenir comple de la conductibilité de la paroi qui les sépare, bien que la conception et la construction de l'appareil soient avant tout déterminées par les coefficients de convection que l'on pense $y$ ' obtenii.

Lorsqu'il s'agit d'un échangeur où l'un des fluides est un gaz, il peut etre nécessaire de tenir compte de la chaleur rayonnée par ce gaz vers la paroi et l'autre lluide, bien que ce soit surtout par convection que s'effectue l'échange de chaleur. Il en seta ainsi chaque fois que la quantité de chaleur transmisc par rayonnement représentera une fraction appréciable de la chaleur échangée par convection.

L'objet de notre exposé est de montrer daus quelles circonstances il peut être utile de s'intéresser ainsi au rayonnement des gaz, el de dire, tres simplement. comment on peal en tenir compte lors du calcul de l'ćchange de chaleur.

\section{II. - NOTIONS GÉNERALES SUR LE RAYONNEMENT}

L'étude des rayonnements énergétiques est très complexe, et il ne saurait être question de l'aborder ici en détail. Aussi, simplifierons-nous à l'extrême, au détriment de la rigueur, et nous bornerons-nous à rappeler quelques principes généraux.

Nous admettrons les lois simples suivantes: - Tout corps rayonne de l'énergie dont la qualité, caractérisée par la longueur d'onde du rayonnement, et la quantité dépendent seulement de la nature de ce corps el de sa température. Si done un corps A rayonne vers un corps $B$ une énergie $E_{A}$ et que le corps $B$ rayonne vers $A$ une ćnergie $\mathrm{E}_{\mathrm{n}}$, la quantité d'énergie « reçue par $\mathrm{A}$ sera $\mathrm{E}_{\mathrm{A}}-\mathrm{E}_{\mathrm{j},}$, énergie effectivement recue si $\mathrm{E}_{\mathrm{S}}$ est supérieure à $\mathrm{E}_{\mathrm{A}}$ ou, au contraire, fournic ì $B$ si $E_{i}$ est plus grand que $E_{1}$.

- On appelle radiance énergétique, ou pouvoir émissif total d'un corps, la quantité $R$ d'énergie que ce corps rayonne, par unité de temps et par unité de surface. La radiance s'exprime, par exemple, en kilocalories $/ \mathrm{m}^{2}$. h.

- On appelle pouvoir absorbant total « $a$ » d'un corps le rapport entre la quantité d'énergie de rayonnement que le corps absorbe et celle qu'il recoit. Le pouvoir « réfléchissant » est évidemment 1 - $a$.

- La loi de Kirchoff exprime que le rapport $\mathrm{R} / \mathrm{a}$ est le même pour tous les corps.

- Par définition, $a$ ne peut pas être supérieur à l'unité.

Le pouvoir absorbant d'un corps réel n'est, en fait, jamais égal à l'unité. On a cependant imaginé un corps théorique pour lequel a serait égal à 1. D'après la loi de Kirchofr, il est évident qu'un tel corps, appelé « corps noir ", est celui dont le pouvoir émissif est maximum. Si l'on appelle $R_{0}$ la radiance du corps noir, on voit que pour un corps réel quelconque :

$$
\frac{R}{a}=\frac{R_{\| 1}}{l}=R_{i n}
$$

- Bien que le corps noir soit de définition yurement théorique, il est possible de realiser expérimentalement un eorps noir presque parfait, et d'étudier les lois qui régissent son rayonnement. La loi de Kirchoff, une fois les lois du corps noir connues, permel de prévoir le comportement des corps reis, a condition de connatue la valeur de leur pouvoir absorbant $a$.

- Lá loi de Stefan-Boltzmann est la lori fondamenlale qui décrit le rayonnement du corps noir : elle peut s'ćnoncer ainsi :

L'énergie lotale rayonnée dans l'unilé de temps, dans tout l'espace, par l'unite do surface du corps noir est proportionnelle à la quatrieme fuissance fo la température absolue.

On écrira donc:

$$
\mathbf{P}_{i 1}=\sigma_{k,} \mathbf{T}^{*}
$$

où $\mathrm{T}$ représente ha lemperralure absolue el o $\sigma_{4}$ une constante, dite * constante de Stéfan-Boltzmann $\%$.

Si l'on exprime $R_{b}$ en kilocalories $/ \mathrm{m}^{2}$. h et $\mathrm{T}$ en degrés Kelvin, ou absolus, la valeur de $\sigma_{0}$ qui differe legerenent suivanl les auteurs, est:

$$
a_{f p}=4,92.10^{-40} \mathrm{lical} / \mathrm{m}^{2} \cdot \mathrm{h} \cdot(\mathrm{K})^{ \pm}
$$

Dans la pratique, pour éviter lemploi des nombres élevés que sont les valeurs de $\mathrm{T}$, on corit de préférence la loi de Stéfan sous la forme:

$$
\left.R_{0}=4,9^{2}\right)\left(\frac{T}{100}\right)^{4} k c_{3} 1 / \mathrm{m}^{2}{ }_{*} h
$$




\section{III. - RAYONNEMENT DES SOLIDES}

Si les solides considérés sont des corps noirs, le calcul de la chaleur échangée entre deux, ou plusieurs de ces corps, est de principe relativement simple. I.es radiances de chacun des corps sont, en effet, égales à $\sigma_{0} \mathrm{~T}_{1}{ }^{4}, \sigma_{0} \mathrm{~T}_{2}{ }^{4}$, etc., où $\mathrm{T}_{1}, \mathrm{~T}_{2}$ sont les températures absolues des corps de surface $S_{1}, S_{2} \ldots$ On peut ainsi calculer, dans l'hypothèse où le milieu qui sépare les corps a un pouvoir absorbant nul, la quantité de chaleur reçue par un élément de surface $d \mathrm{~S}_{1}$ du corps $\mathrm{S}_{1}$, en provenance du corps $S_{2}$, la quantité de chaleur recue par un élément $d S_{2}$, en provenance de $S_{1}$, etc. On intègre ensuite ì la totalité des surfaces $S_{1}$ et $S_{2}$ les quantités élémentaires de chaleur échangée. Le résultat final, une fois l'intégration géométrique eflectuée avee plus ou moins de facilité, peut toujours se mettre sous la forme :

$$
\mathrm{Q}=\sigma_{0} \mathrm{~S}_{1} \omega_{1}{ }^{2}\left(\mathrm{~T}_{1}{ }^{4}-\mathrm{T}_{2}{ }^{4}\right)=\sigma_{0} \mathrm{~S}_{2} \mu_{2}{ }^{1}\left(\mathrm{~T}_{1}{ }^{4}-\mathrm{T}_{2^{4}}\right)
$$

où le coefficient $u_{1}{ }^{2}$ (our $\mu_{2}{ }^{2}$ ) tient compte de l'influence des positions et dinensions géométriques des corps $S_{1}$ et $S_{.2}$. Si $T_{1}$ est supérieur à $T_{2}$, $S_{2}$ recoit de la chaleur de $S_{1}$ et inversement.

Fn particulier, si l'un des corps $\left(S_{1}\right)$ est complètement entouré par une enceinte $\left(S_{2}\right), \mu_{1}^{2}$ est égal à 1, et la quantitó de chaleur échangée est :

$$
\mathrm{Q}=\pi_{0} \mathrm{~S}_{1}\left(\mathrm{~T}_{1}{ }^{4}-\mathrm{T}_{2}{ }^{4}\right)
$$

En réalité, aucun corps réel n'est véritablement un corps noir; par contre, beaucoup de corps industriels (rencontrés dans les foyers de chaudic̀res par exemple) sont des corps " gris», dont le coefficient d'absorption est voisin de l'unité.

Dans ce cas on appellera $\sigma_{1} \mathrm{~T}_{1}{ }^{4}, \sigma_{2} \mathrm{~T}_{2}{ }^{4}$ les radiances des corps (1) et (2), définies d'après la loi de Kirchoff par :

$$
\frac{\sigma_{1}}{a^{1}}=\frac{\sigma_{2}}{a^{2}} \doteq \sigma_{0}
$$

Le calcul sera conduit de la même manière que dans le cas de corps noirs, en tenant compte simplement du fait qu'une partie du rayonnement est chaque fois réfléchie.

Si l'on considère, par exemple, une enceinte grise $S_{\text {. }}$ contenant un corps gris $S_{1}$, la quantité de chaleur échangée pourra être mise finalement sous la forme

$$
\mathrm{Q}=\frac{\mathrm{S}_{1}\left(\mathrm{~T}_{1}{ }^{4}-\mathrm{T}_{2}{ }^{4}\right)}{\left(1 / \sigma_{1}\right)+\left[\left(1 / \sigma_{2}\right)-\left(1 / \sigma_{0}\right)\right] k\left(\mathrm{~S}_{1} / \mathrm{S}_{2}\right)}
$$

où $\mathrm{k}$ est un facteur compris entre 1 et $S_{2} / S_{1}$, qui dépend de la forme géométrique des corps $S_{1}$ et $\mathrm{S}_{2 \mathrm{i}}$.

Sì l’on néglige la chaleur rélléchie, ec qui est justifié lorsque $\sigma_{1}$ et $\sigma_{2}$ sont assez voisins de $\sigma_{4}$, l'expression prend la forme :

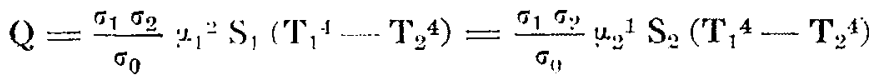

où $y^{2}$ est le coefficient géométrique défini pour les corps noirs. Si, de plus, $T_{2}$ est nettement inférieur à $\mathrm{T}_{1}$, le terme $\mathrm{T}_{1}{ }^{4}-\mathrm{T}_{2}{ }^{4}$ est peu différent de $\mathrm{T}_{1}^{4}$.

Pour les corps gris industriels, on peut admettre sans trop d'erreur que leurs facteurs d'émission, $\sigma_{1} / \sigma_{n}, \sigma_{2} / \sigma_{0}, \ldots$ sont indépendants à la fois de la température et de la longueur d'onde du rayonnement. Cette hypothèse simplifie les calculs, car la radiance d'un tel corps est, dans ce cas, tonjours égale à une fraction bien déterminée de la radiance d'un corps noir de même température.

Signalons enfin que, contrairement à ce qui se passe, par hypothèse, pour un corps noir, la température $\left(\mathbf{T}_{1}, \mathbf{T}_{2}\right)$ d'un corps réel gris peut ne pas être la même sur toute sa surface. Dans ce cas, il suffit, en principe, de diviser les corps qui rayonnent en zones élémentaires où l'on puisse admettre la constance de la température, puis de calculer les échanges de chaleur entrc tous les groupements possibles de ces zones.

\section{IV. - RAYONNEMENT DES GAZ}

Les gaz diffèrent beaucoup du corps noir, et des corps gris industriels, du fait qu'il n'est plus du tout possible d'admettre que leur pouvoir absorbant est constant quelle que soit la Jongueur d'onde du rayonnement.

Pour heaucoup de gaz, toutefois, cette compli- cation apparente conduit à une simplification, en ce sens que leur pouvoir absorbant est nul, ou quasi nul, et qu'on peut, dans tous les cas, le négliger.

C'est le cas de l'azote, de l'hydrogìne, de l'oxygène et de l'air, qui ne présentent pratiquement 
aucune absorption dans le domaine des longueurs d'ondes «thermiques 》 et peuvent être considérés comme parfaitement transparents, incapables de céder ou d'acquérir de la chaleur par rayonnement.

Parmi les gaz habituellement rencontrés dans les installations industrielles, ce sont surtout l'anhydride carbonique et la vapeur d'eau qu'il faut considérer. D'autres gaz tels que l'oxyde de carbone, les hydrocarbures, l'anhydride sulfureux ont des pouvoirs absorbants non négligeables, mais sont en si petite quantité dans les produits de combustion des chaudières à charbon ou à fuel-oil, auxquels nous nous intéressons, qu'il est tout à fait légitime de les négliger.

Pour le $\mathrm{CO}_{2}$, ou la vapeur d'eau, on montre que le pouvoir absorbant est nul pour la plupart des valeurs des longueurs d'onde, mais devient notable pour quelques valeurs bien définies de ces longueurs d'ondes (absorption en bandes).

Le gaz carbonique présente trois handes d'absorption au voisinage des longuenrs d'onde $2,75-4,30$ et 15 microns. Son pouvoir absorbant atteint 1 pour les pointes des bandes à 4,30 et 15 microns. La vapeur d'eau a un spectre d'absorption très compliqué, mais que l'on peut simplifier en un spectre à 5 bandes.

Pour chacune de ces bandes on peut calculer, à chaque température, quelle est l'absorption totale du gaz et la rapporter à l'absorption du corps noir. On exprimera ainsi l'absorption et par conséquent le pouvoir émissif, d'après la loi de Kirchoff, en les rapportant aux caractéristiques $\mathrm{du}$ corps noir. Si la température change, cependant, les pouvoirs absorbants des bandes d'absorption des gaz restent pratiquement les mêmes, et pour les mêmes longueurs d'ondes. Au contraire, l'absorption du corps noir varie en fonction de la longueur d'onde, et son spectre d'absorption varie en fonction de la température (loi de Planck).

On conçoit donc bien que, pour chaque température, on obtiendra un facteur d'émission du gaz, par rapport au corps noir, qui varicra, du fait que la position relative du spectre d'absorption gazeuse et du spectre du corps noir varie en fonction de la température.

D'autre part, le gaz ayant un pouvoir absorbant relativement faible, on peut prévoir que l'absorption dans une masse gazeuse donnée dépendra notablement des dimensions de celle masse gazeuse.

Finalement, on admet généralement les lois suivantes :

- L'absorption ne dépend que du produit de la pression partielle du gaz par l'épaisseur de la couche de gaz, et non pas des rariations séparées de cette pression et de cette épaisseur. Plusieurs expérimentateurs ont discerné que cette loi, qui est bien vérifiée pour le gaz carbonique, ne l'était pas pour la vapeur d'eau. Cependant, les conclusions auxquelles ils arrivent diffèrent quantitativement, bien que tous admettent que, pour la vapeur d'eau, l'absorption dépende aussi de la pression partielle considérée seule. Nous admettrons, pour simplifier, la validité, pour les deux gaz, de la loi énoncée.

- Au voisinage d'une longueur d'onde donnée, l'absorption varie exponentiellement en fonction de l'épaisseur traversée.

En utilisant ces deux lois et en faisant certaines hypotheses simplificatrices sur la forme des bandes d'absorption du gaz et sur la forme du spectre d'émission du corps noir, on arrive finalement à définir :

- Le facteur total d'émission d'une couche d'épaisseur infinie de $\mathrm{CO}_{2}$ ou de $\mathrm{H}_{2} \mathrm{O}$ pur. On exprime ainsi la radiance de la couche de gaz sous forme d'une fraction de la radiance du corps noir considéré à la même température.

La table de la figure 1 donne le résultal d'un tel calcul, en même temps que la radiance $\sigma_{0} \mathrm{~T}^{4}$ du corps noir.

Factenrs d'émission totanx du gaz carbonique el de la vapeur d'eau en couches d'épaisseur infinie.

\begin{tabular}{|c|c|c|c|}
\hline $\begin{array}{l}\text { Température } \\
{ }^{\circ} \mathrm{C}\end{array}$ & $\begin{array}{l}\text { Radiance } \\
\text { totale } \\
\text { du } \\
\text { corps noir } \\
\text { keal } / \mathrm{m}^{2} . \mathrm{h}\end{array}$ & $\begin{array}{c}\text { Facleur } \\
\text { d'ćmission } \\
\text { du gaz } \\
\text { carbonique } \\
(\%)\end{array}$ & $\begin{array}{c}\text { Facteur } \\
\text { d'émission } \\
\text { de lal } \\
\text { vapeur d'eau } \\
(\%)\end{array}$ \\
\hline 200 & 2.460 & 120 & 36.0 \\
\hline 300 & 5.300 & 12,0 & 28,0 \\
\hline 400 & 10.090 & 12,0 & 24,0 \\
\hline 500 & 17.560 & 12,0 & 21,7 \\
\hline 600 & 28.580 & 11,9 & 19,7 \\
\hline 700 & 44.100 & 11,8 & 18,0 \\
\hline 800 & 65.200 & 11,6 & 16,6 \\
\hline 900 & 93.100 & 11,3 & 15,5 \\
\hline 1.000 & 129.200 & 11,0 & 14,5 \\
\hline 1.100 & 174.800 & 10,5 & 13,5 \\
\hline 1.200 & 232.000 & 10,0 & 12,6 \\
\hline 1.300 & 301.000 & 9,5 & 11,8 \\
\hline 1.400 & 385.000 & 9,0 & 11,0 \\
\hline 1.500 & $486.000)$ & 8,5 & 10,3 \\
\hline 1.600 & $80(0.0000)$ & 8,0 & 9,6 \\
\hline 1.700 & 746.000 & 7,5 & 9,0 \\
\hline 1.800 & 909.000 & 6,9 & 8,3 \\
\hline
\end{tabular}

Frf. 1

- Le facteur total d'imission d'une couche d'épaisseur finie de $\mathrm{CO}_{2}$ ou de $\mathrm{H}_{2} \mathrm{O}$. On notera que, dans ce cas, ec n'est pas l'épaisscur réelle de la couche que l'on considère, mais le produit de cette épaisscur par la pression partielle. On notera, d'autre part, que l'absorption (et l'émis- 
sion) ne sont pas exactement des fonctions exponentielles de l'épaisseur ainsi définie, bien que l'on ait admis une loi exponentielle d'absorption à une longueur d'onde donnée. Ceci provient de l'adoption des hypothèses dont nous avons parlé

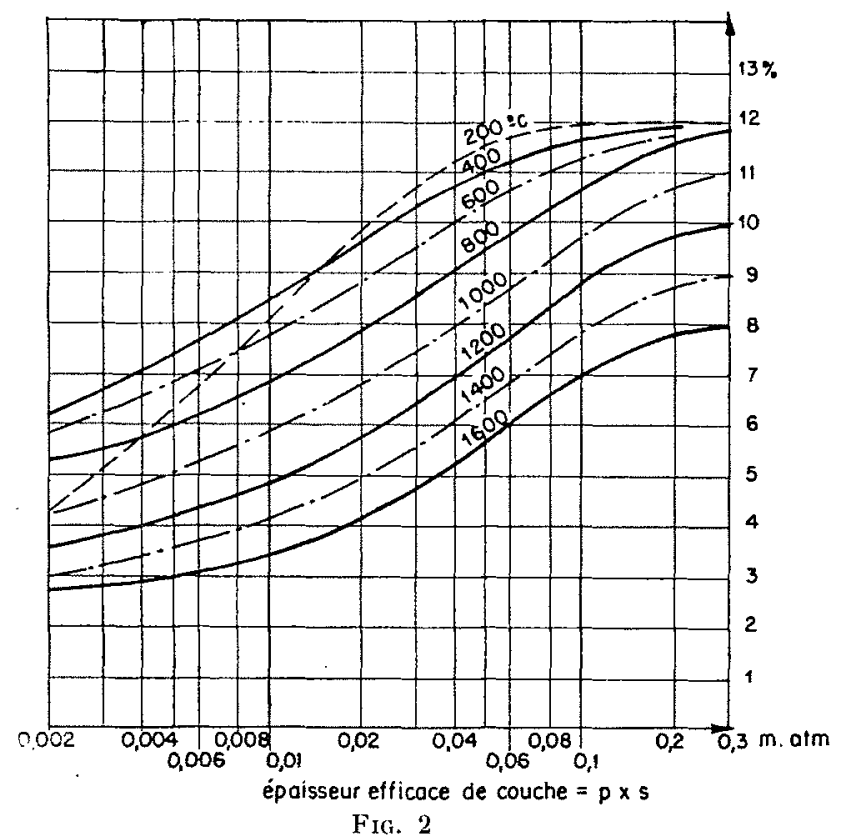

Rayonnement des couches de $\mathrm{CO}_{2}$ en pour cent du rayounement du corps noir, en fonction de la température en " $\mathrm{C}$ et de l'épaisseur efficace de couche en m. atm.

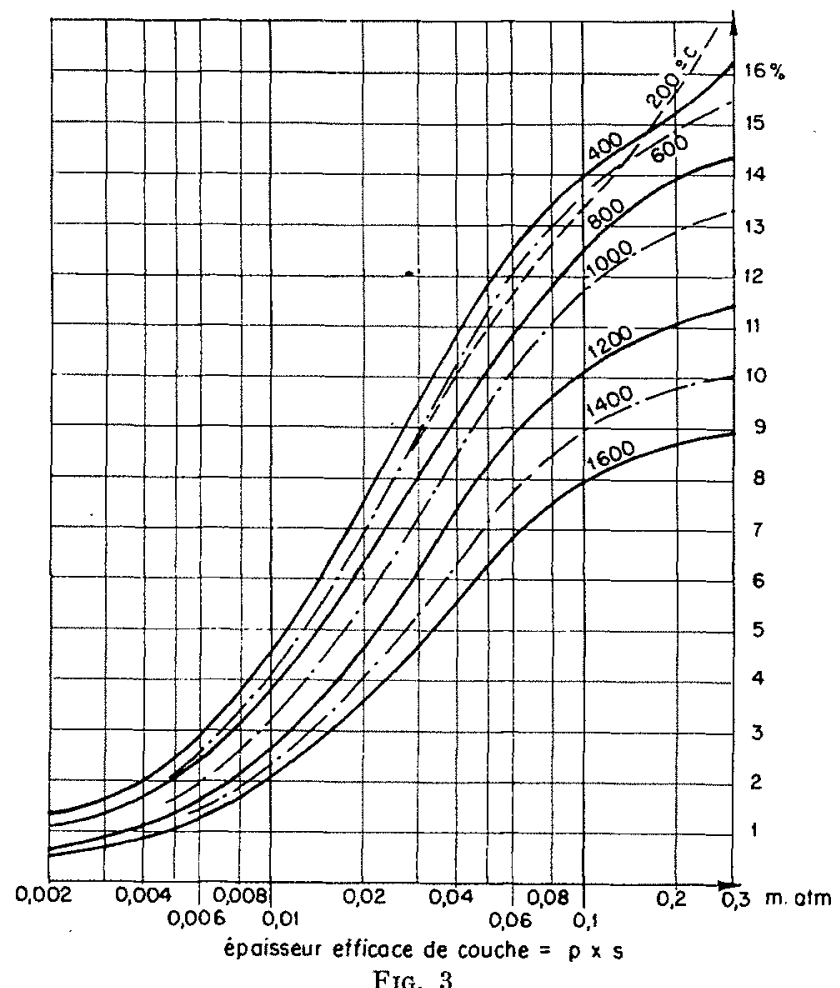

Rayonnement des couches de vapeur d'eau en pour cent cu rayonnement du corps noir, en fonction de la température en "C et de l'épaisseur effieace de couche en m. atm. précédemment à propos de la forme des bandes du gaz et du spectre du corps noir. Les abaques des figures 2 et 3 ont été établis ainsi pour le $\mathrm{CO}_{2}$ et la vapeur d'eau.

- Pour obtenir des abaques d'emploi pratique, et suffisamment approchés dans la plupart des cas usuels, on peut, puisqu'il s'agit en dérinitive d'examiner l'influence du rayonnement des gaz dans les parcours de fumées dans les échangeurs de chaleur, calculer une pression partielle du gaz qui corresponde à des conditions habituelles de combustion, et ne conserver comme paramètre que l'épaisseur réelle de couche rayonnante. On peut, en outre, au lieu d'exprimer les facteurs d'émission, calculer directement la radiance de la couche gazeuse. C'est ainsi (fu'ont été établis les graphiques $n^{\circ} 4$ et 5 .

Lorsque le $\mathrm{CO}_{2}$ et la vapeur d'ean sont présents simultanément dans les gaz de combustion, ce qui est, en pratique, toujours le cas, on ajoutera purement et simplement les quantités de chaleur rayonnées par chacun des gaz. Certains auteurs pensent que l'émission, au total, est dans ce cas supérieure à la somme des deux émissions, et certains autres auteurs pensent, au contraire, qu'elle est plus faible. Dans tous les cas cependant, l'on s'accorde à penser que l'émission est, au fotal, peu différente de la somme des deux émissions, ce qui justifie notre simplification.

- Pour calculer la quantité de chaleur échangée par rayonnement entre une couche gazeuse et les parois solides qui l'entourent, il faudrait, en principe, faire intervenir le pouvoir absorbant de cette paroi. En pratique; il est suffisant d'utiliser pour cette valeur le facteur d'émission du gaz lui-même, considéré à la température de la paroi, du fait que la paroi ne recoit que des rayons dont la longueur d'onde correspond aux bandes d'absorption du gaz.

- De la sorte, pour des gaz entourant une surface, on écrira très simplement la quantité de chaleur cchangée par rayonnement, rapportéc à l'unité de surface de paroi, sous la forme:

$$
\mathrm{Q}=\sigma_{g} \mathrm{~T}_{g}{ }^{4}-\sigma_{p} \mathrm{~T}_{p}{ }^{4}
$$

où $\mathrm{T}_{g j}$ et $\mathrm{T}_{p}$ sont les températures du gaz et de la paroi, et $\sigma_{g}$ et $\sigma_{p}$ les radiances du gaz à sa propre température et à celle de la paroi.

Si l'on appelle $\varepsilon$ le facteur d'émission défini par $\approx=\left(\sigma / \sigma_{01}\right)$, sì $\sigma$ et $\sigma_{0}$ sont la constante du gaz et celle du corps noir, on pourra écrire encore :

$$
\mathrm{Q}=\sigma_{0}\left(\varepsilon_{\eta} \mathrm{T}_{\eta}^{4}-\varepsilon_{p} \mathrm{~T}_{\eta}^{4}\right)
$$

c'est-à-dire :

$$
\mathrm{Q}=4,92\left[\varepsilon_{i ;}\left(\frac{\mathrm{T}_{g}}{100}\right)^{4}-\varepsilon_{p}\left(\frac{{ }^{\mathrm{T}} \mathrm{T}_{p}}{100}\right)^{4}\right] \mathrm{kcal} / \mathrm{h} . \mathrm{m}^{2} \text {. }
$$


- Si l'on veut comparer l'ordre de grandeur des énergies rayonnées et des énergies transmises par convection par exemple, on pourra définir un « coefficient de rayonnement », $\alpha_{r}$, par la fornule :

$$
\alpha_{r}=\frac{\sigma_{0}\left(\varepsilon_{g} \mathrm{~T}_{g}^{4}-\varepsilon_{p} \mathrm{~T}_{p}{ }^{4}\right)}{\mathrm{T}_{g}-\mathrm{T}_{p}}
$$

Ce coefficient $\alpha_{i}$, doit être ajouté au coefficient externe de convection, le gaz étant supposé entourer les surfaces d'échange, lors du calcul du coefficient global de transmission. Au contraire, si le gaz rayonnant circule à l'intérieur des tubes du faisceau d'échange, c'est au coelficient interne que $\alpha_{\text {. }}$. doit être ajouté.

- Si enfin, ainsi qu'il se produit souvent, la température du gaz et celle de la paroi varient continûment dans un faisceau d'échange, il sera nécessaire, soit de diviser l'échangeur en zones élémentaires oì les tempéralures soient quasi constantes, d'y calculer les quantites de chaleur rayonnées et de les sommer finalement, soit, pour simplifier, d'admeltre une valcur moyenne de la température des gaz et de la température des parois.

\section{V. - APPLICATIONS PRATIQUES}

\section{1. - Chambres de combustion}

Dans les chambres de combustion qui brûlent du charbon pulvérisé ou du fuel-oil, le $\mathrm{CO}_{2}$ et la vapeur d'eau présents rayonnent, mais la quanfité d'énergie rayonnée par les gaz eux-mèmes est négligeable par rapport à celle rayonnée par les corpuscules de la flamme: grains de charbon ou gouttes très petites de fuel-oil en comhustion.

Le facteur d'émission des gaz contenus dans la chambre de combustion est de quelques pour cent, alors que la radiance des "flammes " peut atteindre les $9 / 10$ de celle du corps noir.

C'est pour cette raison que le ponvoir rayonnont des flammes de fuel-oil est, généralement, supérienr a celui des flammes de charbon, à température égale, du fait que les points matériels rayonnants y sont plus nombreux; présenlant donc une surface rayonnante considérable.

\section{2. - Rayonnement propre des gaz}

Par contre, dans les échangeurs situés en aval de la chambre de combustion, les particules de charbon et de cendres, ou les gouttelettes de fuel-oil, se sont à proprement parler volatilisées ou bien, s'il s'agit des cendres, déposées au cendrier. De sorte que ce sont bien les gaz cuxmêmes, $\mathrm{CO}_{2}$ et vapeur d'cau, qui restent capables de rayonner.

A titre d'exemple, nous pouvons calculer quelle sera, du fait de la plus grande proportion d'hydrogène que contient le combustible, l'excès de la quantité de chaleur rayonnée par des fumées de fuel-oil sur celle rayonnce par des fumées de charbon.

On considère un fuel-oil de P.C.I. 9.700 kcal/kg, ne contenant pas d'ean et d'une teneur en hydrogène de $11,5 \%$.

La masse d'eau formée par combinaison de l'hydrogène du combustible avec l'oxygène de l'air comburant est de $1,035 \mathrm{~kg}$ par kilogramme de combustible, et le volume de vapeur d'eau

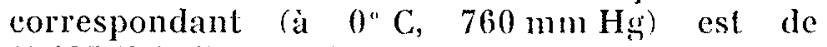
$(1,035 / 0,804)=1,287 \mathrm{~m}^{3} \mathrm{~N}$.

Si on brûle ce combustible avec un excès d'air de $20 \%$, les fumées réelles occupent, par lig de fuel-oil brûlé, un volume de $13,25 \mathrm{~m}^{3} \mathrm{~N}$, de sorte que, dans les fumees, la pression particlle de la rapeur d'eau est de $(1,287 / 13,25)=0,0973$. Simultanément, on peut calculer que la teneur des fumées humides en gaz carbonique est de 11,4 of.

$\mathrm{Si}$ on considere maintenant un charbon "moyen , contenant $8 \%$ d'eau, $16 \%$ de stériles, $65 \%$ de carbone, $4,5 \%$ d'hydrogène et $6 \%$ d'oxygène, de P.C.I. 6280, el qu'on le brûle avec un exces d'air tel que la teneur en $\mathrm{CO}$ des fumées totales soit également de $11,4 \%$, on calcule que la pression parlielle de la vapeur d'eau sera seulement de 0,0 ó 68 .

Pour une même épaissenr de conche de saz, le rayonnement des fumces de fucl-oil sera supérieur à celui des fumées de charbon.

Si, pour fixer les idces, on s'intéresse à la radiance des fumées en ce point de parcours oi elles sont à $6000^{\circ} \mathrm{C}$ et si l'on suppose que la couche réelle de saz a $100 \mathrm{~mm}$ d'épaisseur, lo produit de la pression partielle par l'épaisscur sera :

- Pour le CO., quel que soil le combustible : 0,0114 ef sa radiance à $6000^{\circ} C$ sera 0,079 fois celle du corps noir.

- Pour la vapeur d'eau dans les fumćes du fueloil, le produit de la pression partielle par l'épaisseur de couche sera 0,00973 et la jadiance $0,0,38$ fois celle du corps noir. 
Dans les fumées de charbon, les valeurs correspondantes seront 0,00568 et 0,022 .

De sorte que le rayonnement du gaz sera, au tolal, pour les fumées de fuel-oil :

$$
7,9+3,8=11,7 \%
$$

du rayormement du corps noir.

Pour les fumces de charbon:

$$
7,9+2,2,=10,1 \%
$$

du rayonnement du corps noir.

L'écart entre les deux radiances est donc, dans ce cas, de l'ordre de $15 \%$.

\section{3. - Comparaison avec l'énergie transmise par convection}

Comme nous l'avons déjà indiqué dans l'introduction, le calcul de l'énergie transmise par le rayonnement des gaz n'a d'intérêt que si la quantité de chaleur transmise par rayonnement représente une fraction appréciable de la chaleur transmise par convection.

Pour montrer que ce peut être le cas, considérons un échangeur à tubes d'eau, parcouru par des fumées de fuel-oil. Admettons que l'épaisseur de couche soil de $100 \mathrm{~mm}$ et supposons comme précédemment que la pression partielle du CO, dans les fumées soit 0,114 et celle de la vapeur d'eau de 0,0973 . Supposons, de plus, que la température de paroi soit de $240^{\circ} \mathrm{C}$.

On calculera la quantité de chaleur recue par unité de surface de paroi par la formule indiquée précédemment :

$$
\mathrm{Q}=4,92\left[\varepsilon_{g}\left(\frac{\mathrm{T}_{g}}{100}\right)^{4}-\varepsilon_{p}\left(\frac{\mathrm{T}_{p}}{100}\right)^{1}\right] \mathrm{kcal} / \mathrm{h} \cdot \mathrm{m}^{2}
$$

Les valeurs des facteurs d'émission $\varepsilon_{y}$ et $\varepsilon_{p}$ seront obtenues en faisant la somme des facteurs propres au $\mathrm{CO}_{2}$ et à la vapeur d'eau.

En supposant que les fumées aient une température $\mathrm{T}_{g}$ de $400^{\circ} \mathrm{C}, 6000^{\circ} \mathrm{C}, 800^{\circ} \mathrm{C}$ et $1.000^{\circ} \mathrm{C}$ successivement, on lit sur les abaques $n^{1 / 2} 2$ et 3 les valeurs suivantes:

\begin{tabular}{|c|c|c|c|c|c|}
\hline Température & $240^{\circ} \mathrm{C}$ & $400^{\circ} \mathrm{C}$ & $600^{\circ} \mathrm{C}$ & $800^{\circ} \mathrm{C}$ & $1000^{\circ} \mathrm{C} \mid$ \\
\hline$\varepsilon$ gaz carbonique. & 0,085 & 0,087 & 0,079 & 0,070 & 0,059 \\
\& vapeur d'eau..... & 0,040 & 0,045 & 0,638 & 0,036 & 0,030 \\
₹ au total ......... & 0,125 & 0,132 & 0,117 & 0,106 & 0,089 \\
\hline
\end{tabular}

En reportant les valeurs de $\varepsilon$, ' $\mathrm{T}_{g}$ et $\mathrm{T}_{p}$ dans la formule et en effectuant, on obtient les quantités de chaleur, par unité de surface, suivantes :

$$
\begin{array}{rl}
\mathrm{T}_{g}=400^{\circ} \mathrm{C} & \mathrm{Q}=910 \mathrm{kcal} / \mathrm{m}^{2} . \mathrm{h} \\
\mathrm{T}_{g}=600^{\circ} \mathrm{C} & \mathrm{Q}=2.920- \\
\mathrm{T}_{o}=800^{\circ} \mathrm{C} & \mathrm{Q}=6.490- \\
\mathrm{T}_{g}=1.000^{\circ} \mathrm{C} & \mathrm{Q}=11.070
\end{array}
$$

(On aurait pu obtenir directement ces résultats en utilisant les abaques $\mathrm{n}^{\circ *} 4$ et 5 établis pour des fumées ayant justement la composition envisagée.)

Si l'on veut comparer ces quantités de chaleur à celles échangées par convection, on calcule le «coefficient de rayonnement $\alpha_{r}$ » :

$$
\alpha_{r}=\frac{\mathbf{Q}}{\mathrm{T}_{q}-\mathbf{T}_{z}}
$$

Les valeurs obtenues sont :

$$
\begin{aligned}
& \mathrm{T}_{g}=400^{\circ} \mathrm{C}, \alpha_{r}=6 \mathrm{kcal} / \mathrm{m}^{2} \cdot \mathrm{h}^{\circ} \mathrm{C} \\
& \mathrm{T}_{g}=600^{\circ} \mathrm{C}, \alpha_{r}=8 \quad- \\
& \mathrm{T}_{g}=800^{\circ} \mathrm{C}, \alpha_{r}=11 \\
& \mathrm{~T}_{g}=1.000^{\circ} \mathrm{C}, \alpha_{r}=15
\end{aligned}
$$

L'on sait, par ailleurs, que le coefficient de convection externe dans des échangeurs de ce genre, est d'un ordre de grandeur compris entre 25 et $50 \mathrm{kcal} / \mathrm{m}^{2} . \mathrm{h}{ }^{\circ} \mathrm{C}$.

Le coefficient de rayonnement n'est donc pas du tout négligeable, dans ce cas, vis-d்-vis de l'échange par convection, même si la température des gaz est relativement basse. A $400^{\circ} \mathrm{C}$, le rayonnement contribue déjà pour 15 à $25 \%$ à l'échange de chaleur.

La chaleur échangée par rayomnement ne dépend guère que de l'épaisseur de couche et de la pression partielle du gaz, tant que celui-ci entoure complètement les parois absorbantes. Aussi, des faisceaux tubulaires disposés différemment recevront-ils la même quantité de chaleur de rayonnement si l'épaisseur de couche est la même, ainsi quc la nature des gaz, tandis que le coefficient de convection peut être notablement modifié, dans les mêmes conditions, par le changement de disposition des bancs de tubes. 
Le présent abaque est établi pour les fumées d'un fuel-oil lourd $\mathrm{n}^{\circ} 2$, de composition moyenne:

P.C.S. : $10.350 \mathrm{kcal} / \mathrm{kg}$; P.C.I. : $9.700 \mathrm{kcal} / \mathrm{kg}$ brûlé avec un excès d'air de 1,2 .

Volume d'air de combustion. . . . . . $12,58 \mathrm{Nm}^{3} / \mathrm{kg}$

Volume des fumées sèches.......... $11,95 \mathrm{Nm}^{3} / \mathrm{kg}$

Volume des fumées humides (réelles) ... $13,25 \mathrm{Nm}^{31} / \mathrm{kg}$

$\mathrm{CO}_{2}$ apparent (ORSAT) $\ldots \ldots \ldots \ldots \ldots \ldots, 12,6 \%$

$\mathrm{CO}_{2}$ réel (sur fumées humides)........ 11,4\%

Teneur en humidité des fumées...... $9,73 \%$

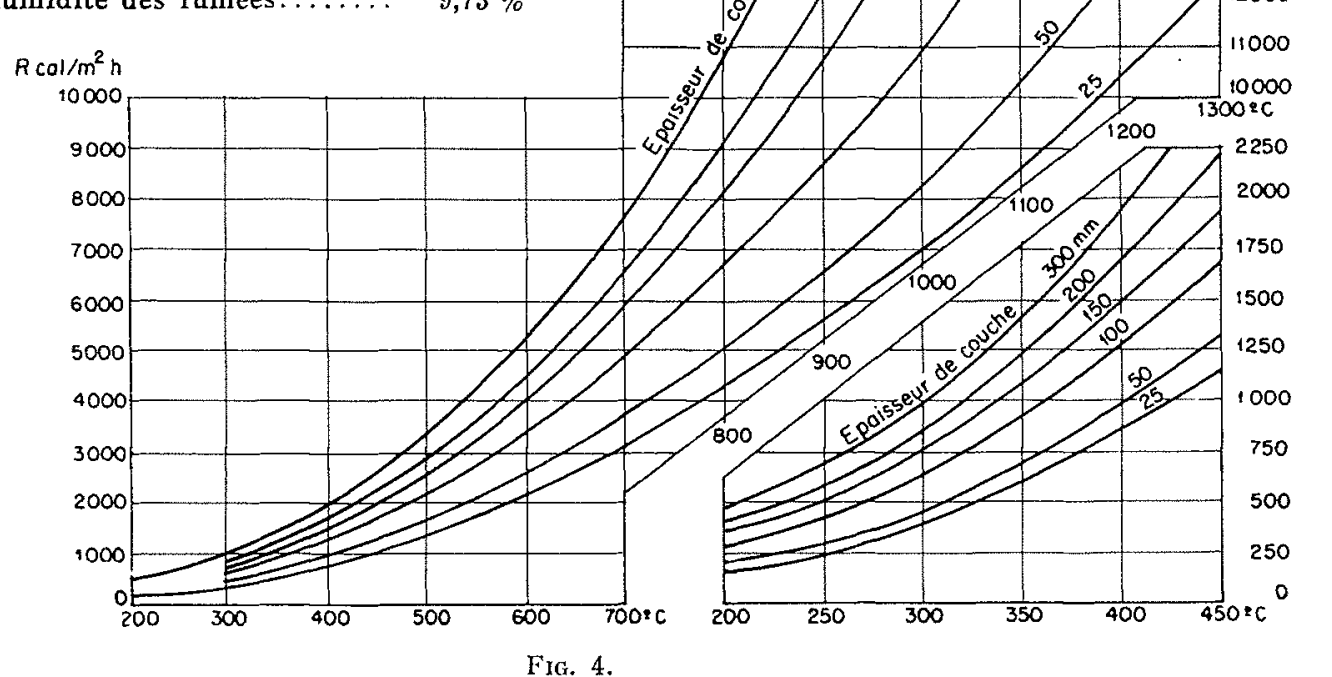

FIG. 4.

Rayomement des fumées de fuel-oil à la pression atmosphérique.

Faibles épaisseurs de couche.

Le présent abaque est établi pour les fumées d'un fuel-oil lourd $n^{\circ} 2$, de composition moyenne :

P.C.S. : $10.350 \mathrm{kcal} / \mathrm{kg} ;$ P.C.I. : $9.700 \mathrm{kcal} / \mathrm{kg}$ brûlé avec un excès d'air de 1,2 .

Volume d'air de combustion ........ $12,58 \mathrm{Nm}^{3 /} / \mathrm{kgg}$

Volume des fumées sèches........... 11,95 Nm3/

Volume des fumées humides (réelles) .. $13,25 \mathrm{Nm}^{3} / \mathrm{kg}$

$\mathrm{CO}_{2}$ apparent (ORSAT) $\ldots \ldots \ldots \ldots \ldots \ldots, 12,6 \%$

$\mathrm{CO}_{2}$ réel (sur fumées humides) ....... 11,4 \%

Teneur en humidité des fumées...... $9,73 \%$

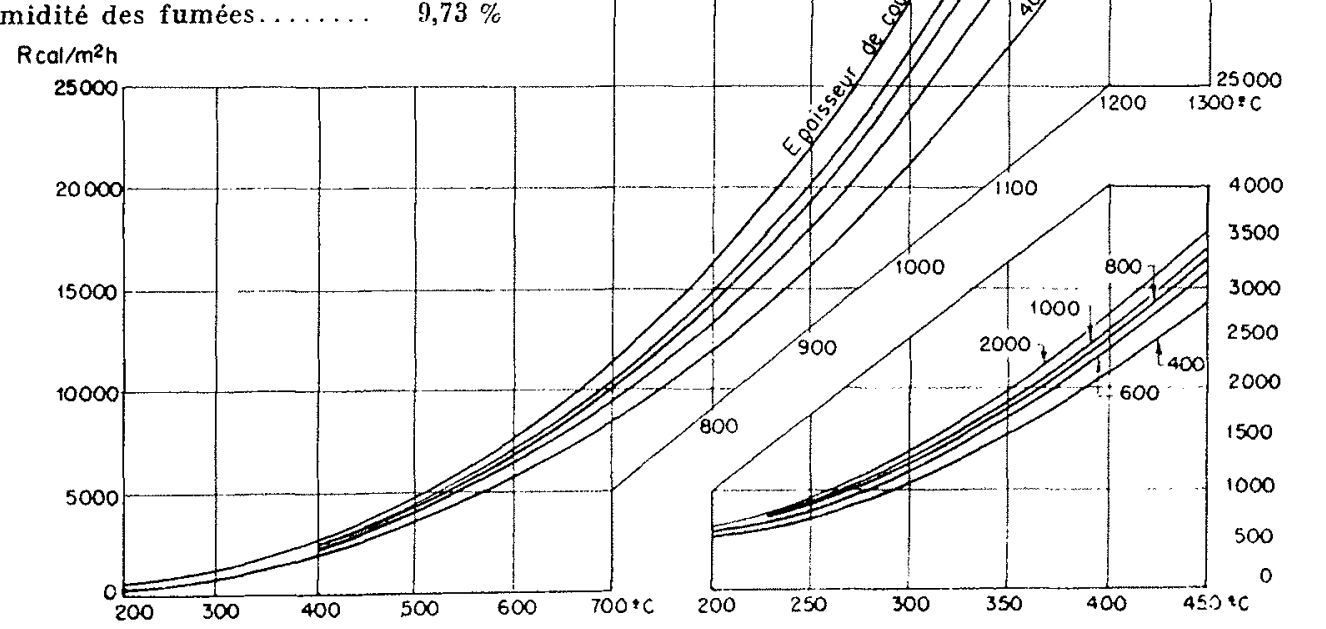

FIG. 5

Rayonnement des fumces de fuel-oil a la pression atmosphérique.

Fortes épaisseurs de couche. 


\section{4. - Calcul de l'épaisseur de couche}

I.e calcul exact de l'épaisseur de couche à introduire dans le calcul du facteur d'émission dans chaque disposition géométrique particulière est relativement compliqué.

Certains auteurs ont cependant publié des tables qui indiquent quelle est la relation entre une dimension caractéristique du système et l'épaisseur utile de couche.

Lorsqu'il s'agit par exemple de faisceaux de tubes parallèles régulièrement espacés, baignés par un gaz qui rayonne, les épaisseurs utiles de couches, dans quelques cas de géométrie simple, sont les suivantes (on appelle $p$ la distance qui sépare les parois de deux tubes voisins, $p$ est donc égale à la distance entre axes diminués du diamètre $d$ des tubes):

\begin{tabular}{|c|c|c|}
\hline & $\begin{array}{c}\text { Dimension } \\
\text { caractéris- } \\
\text { tique }\end{array}$ & $\begin{array}{c}\text { Epaisseur } \\
\text { de } \\
\text { couche }\end{array}$ \\
\hline $\begin{array}{c}\text { Faisceau de tubes en réseau } \\
\text { triangulaire équilatéral, } \\
\text { où } p=d \ldots \ldots \ldots \ldots \ldots \ldots\end{array}$ & $p$ & $2,8 p$ \\
$\begin{array}{c}\text { Faisceau de tubes en réseaun } \\
\text { triangulaire équilatéral, } \\
\text { où } p=2 d \ldots \ldots \ldots \ldots\end{array}$ & $p$ & $3,8 p$ \\
$\begin{array}{c}\text { Da même, mais } p=3 d \ldots \ldots \\
\text { Faisceau de tubes en réseau } \\
\text { carré, où } p=d \ldots \ldots \ldots\end{array}$ & $p$ & $4,3 p$ \\
\hline
\end{tabular}

\section{I S C US SION}

présidenl: M. GIBRaT

M. le Présidenl renercie M. Storz et souligne l'intérêt du mémoire de MM. VIgNeron et STolz: l'ctude du rayomnement à très hiute température montre que, daus le phénomène d'échange de chaleur, interviennent des facteurs differrents de ceux qui, comme la convection, s'apparentent à la mécanique des fluides.

M. Orrvrer estime que la transmission de chalcur aux gaz par rayonnement est une question qui exige encore une importante mise au point; ses difficultés sont de deux ordres :

D'une part, à la traversée d'une couche mince, il n'y a pas seulement affaiblissement de lat brillauce énergétique : il y a renforcement par la rémission de tous les éléments du gaz; on trouve donc des équations intégrodifr'érentielles.

D'autre part, et c'est beaucoup plus grave, la loi d'affaiblissement varie selon la longueur d'onde, d'une façon extrêmement fine pour la vapeur d'eau (Mijoite, Bruxelles, mesures faites à la Jungfrau).

De ce fait, il est très dangereux de prendre unc loi moyenne d'affaiblisșement - qui serait exponentielle sur une bande de longueur d'onde d'étendue appréciable. On ne peut espérer - au bout de calculs consiclérables - qu'une approximation incertaine.

M. LE Foll fait remarquer que le rayonnement des gaz est un facteur favorable du point de vue thermodynamique, surtout pour des températures de l'ordre de $1000^{\circ}$ absolus et des pressions nettement différentes de la pression atmosphérique, qui seront celles des futurs réacteurs nucléaires.

Dans ces conditions de température et de pression, la loi de proportionnalité à l'épaisseur fictive de couche, valable pour les chaudières classiques, ne s'applique peut-être plus. MM. Stozz et LE FoLL sont cependant d'accord pour constater l'intérêt de calculs d'ordre de grandeur concernant l'influence du rayonnement du gaz carbonique sur les échanges de chaleur dans les futurs réacteurs.

M. Forrier confirme qu'il ne faut pas négliger les quantités de chaleur échangées par rayonnement des saz: dans un échangeur constitué par un tuyau central chauffé à $400^{\circ}$ absolus par des gaz de combustion et entouxé d'un conduit circulaire concentrique et calorifugé (suivant schéma ci-dessous), il a constaté que la courbe de

\section{alr extérieur}

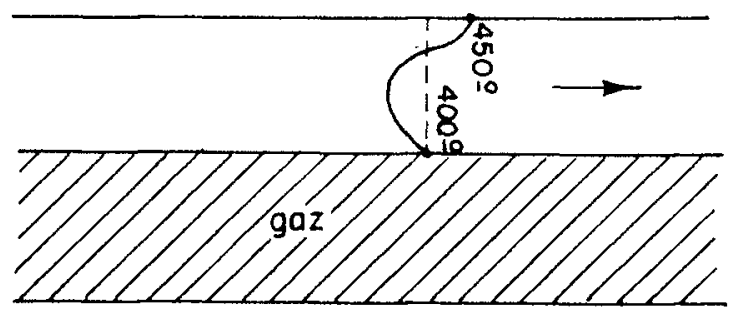

température présentait un minimum au centre et que la paroi extérieure était beaucoup plus chande - de $50^{\circ} \mathrm{C}$ environ - que le gaz intérieur; cela semble dû à ce que la paroi extérieure absorbe une quantité de chaleur non négligeable provenant du rayonnement de la paroi intérieure et il faut tenir compte de cette différence de température entre les deux parois dans le calcul des quantités de chaleur entraînées par les gaz. 